\title{
GROUP EXTENSIONS AND THE PRIMITIVE IDEAL SPACES OF TOEPLITZ ALGEBRAS
}

\author{
SRIWULAN ADJI \\ School of Mathematical Sciences, Universiti Sains Malaysia, 11800 Penang, Malaysia \\ e-mail:wulan@cs.usm.my \\ IAIN RAEBURN \\ School of Mathematics and Applied Statistics, University of Wollongong NSW 2522, Australia \\ e-mail:raeburn@uow.edu.au \\ and RIZKY ROSJANUARDI \\ Department of Mathematics, Universitas Pendidikan Indonesia, Jl. Dr. Setia Budhi 229, \\ Bandung 40154, Indonesia \\ e-mail:rizky@upi.edu
}

(Received 24 February, 2006; revised 19 October, 2006; accepted 10 November, 2006)

\begin{abstract}
Let $\Gamma$ be a totally ordered abelian group and $I$ an order ideal in $\Gamma$. We prove a theorem which relates the structure of the Toeplitz algebra $\mathcal{T}(\Gamma)$ to the structure of the Toeplitz algebras $\mathcal{T}(I)$ and $\mathcal{T}(\Gamma / I)$. We then describe the primitive ideal space of the Toeplitz algebra $\mathcal{T}(\Gamma)$ when the set $\Sigma(\Gamma)$ of order ideals in $\Gamma$ is well-ordered, and use this together with our structure theorem to deduce information about the ideal structure of $\mathcal{T}(\Gamma)$ when $0 \rightarrow I \rightarrow \Gamma \rightarrow \Gamma / I \rightarrow 0$ is a non-trivial group extension.
\end{abstract}

2000 Mathematics Subject Classification. 46L55.

Introduction. Let $\Gamma$ be a totally ordered abelian group with positive cone $\Gamma^{+}$, and denote by $\left\{e_{x}: x \in \Gamma^{+}\right\}$the usual basis for the Hilbert space $\ell^{2}\left(\Gamma^{+}\right)$. For each $x \in \Gamma^{+}$, there is an isometry $T_{x}$ on $\ell^{2}\left(\Gamma^{+}\right)$such that $T_{x} e_{y}=e_{x+y}$ for all $y \in \Gamma^{+}$. The Toeplitz algebra of $\Gamma$ is the $C^{*}$-subalgebra $\mathcal{T}(\Gamma)$ of $B\left(\ell^{2}\left(\Gamma^{+}\right)\right)$generated by the isometries $\left\{T_{x}: x \in \Gamma^{+}\right\}$. These Toeplitz algebras include as special cases the algebras studied by Coburn [7] and Douglas [8], and generalisations to various classes of partially ordered groups have attracted a great deal of attention in recent years (see $[12,13,10,11]$, for example).

In [4], we considered the problem of describing the ideal structure of $\mathcal{T}(\Gamma)$, and found that a crucial ingredient is the set $\Sigma(\Gamma)$ of order ideals in $\Gamma$, which is itself totally ordered under inclusion. We showed that the primitive ideals of $\mathcal{T}(\Gamma)$ are parametrised by the disjoint union $X(\Gamma):=\bigsqcup\{\widehat{I}: I \in \Sigma(\Gamma)\}$ of the duals of the discrete abelian

This research was supported by the Australian Research Council and URGE (the Indonesian Research Fund for Doctoral Sandwich Programs). The first author is an associate of the Abdus Salam International Center for Theoretical Physics in Trieste, Italy. Some results in this paper are from the third author's PhD thesis (Institut Teknologi Bandung, Indonesia, 2005). 
groups $I$ [4, Theorem 3.1]. We then sought to describe the topology on $X(\Gamma)$ which corresponds to the hull-kernel topology on the primitive ideal space $\operatorname{Prim} \mathcal{T}(\Gamma)$.

When $\Sigma(\Gamma)$ is order isomorphic to a subset of $\mathbb{N} \cup\{\infty\}$ (for example, if $\Sigma(\Gamma)$ is finite or $\Gamma$ is an anti-lexicographic direct sum over $\mathbb{N}$ ), the appropriate topology on $X(\Gamma)$ is what we shall call here the upwards-looking topology: the closure of a subset $F$ of $X(\Gamma)$ consists of the characters $\gamma \in \widehat{J}$ with the property that, for every open neighbourhood $N$ of $\gamma \in \widehat{J}$, there exist $I \in \Sigma(\Gamma)$ and $\chi \in N$ such that $I \subset J$ and $\left(I,\left.\chi\right|_{I}\right)$ belongs to $F$. This is proved in [4, Proposition 4.7]. Even though we know by example that the upwards-looking topology is not the correct topology for every $\Gamma$ (see [4, Example 4.10]), it seems useful to identify the groups $\Gamma$ for which it is the correct topology, and we do this here.

We begin with a short section in which we set up notation, recall the basic properties of Toeplitz algebras, and describe the parametrisation of $\operatorname{Prim} \mathcal{T}(\Gamma)$ found in $[4, \S 3]$. In $\S 2$, we prove a new version of the structure theorem for the Toeplitz algebras of group extensions $0 \rightarrow I \rightarrow \Gamma \rightarrow \Gamma / I \rightarrow 0$ in which $I$ is an order ideal. This theorem extends a result of Adji [2] which identifies a certain quotient of $\mathcal{T}(\Gamma)$ as an induced algebra with fibre $\mathcal{T}(\Gamma / I)$. Here we show also that the kernel of the quotient map onto the induced algebra is naturally Morita equivalent to the commutator ideal in the Toeplitz algebra $\mathcal{T}(I)$.

Our main theorem, which is the subject of $\S 3$, says that $\operatorname{Prim} \mathcal{T}(\Gamma)$ is homeomorphic to $X(\Gamma)$ with the upwards-looking topology if and only if the totally ordered set $\Sigma(\Gamma)$ is well-ordered in the sense that every non-empty subset has a least element. Our main tools are the structure theorem proved in $\S 2$, and a new general result on the upwardslooking topology (Proposition 3.3). The proof of Proposition 3.3 uses classical Toeplitz operators as well as the universal property of $\mathcal{T}(\Gamma)$ which was the main tool in [4]. The last section combines the results of the previous sections to see what can be said about $\operatorname{Prim} \mathcal{T}(\Gamma)$ when parts of $\Sigma(\Gamma)$ are well-ordered.

1. Toeplitz algebras. Let $\Gamma$ be a totally ordered abelian group with positive cone $\Gamma^{+}=\{x \in \Gamma: x \geq 0\}$. We write $e_{x}$ for the characteristic function of the singleton set $\{x\}$, so that $\left\{e_{x}: x \in \Gamma^{+}\right\}$is the usual orthonormal basis for $\ell^{2}\left(\Gamma^{+}\right)$. For $x \in \Gamma^{+}$, we denote by $T_{x}$ the isometric linear operator on $\ell^{2}\left(\Gamma^{+}\right)$which satisfies $T_{x}\left(e_{y}\right)=e_{x+y}$ for every $y \in \Gamma^{+}$; when there is more than one group around, we write $T_{x}^{\Gamma}$ for emphasis. The Toeplitz algebra of $\Gamma$ is the $C^{*}$-subalgebra $\mathcal{T}(\Gamma)$ of $B\left(\ell^{2}\left(\Gamma^{+}\right)\right)$generated by $\left\{T_{x}\right.$ : $\left.x \in \Gamma^{+}\right\}$.

It is well-known that $\mathcal{T}(\Gamma)$ acts irreducibly on $\ell^{2}\left(\Gamma^{+}\right)$, and this is proved for much more general $\Gamma$ in [12, Theorem 3.13]. Perhaps it is worth pointing out that this is easy to see when $\Gamma$ is totally ordered.

LeMma 1.1. The Toeplitz algebra $\mathcal{T}(\Gamma)$ of a totally ordered abelian group $\Gamma$ acts irreducibly on $\ell^{2}\left(\Gamma^{+}\right)$.

Proof. Suppose that $P$ is a projection in $\mathcal{T}(\Gamma)^{\prime}$ and $x \in \Gamma^{+}$. For every other $y \in \Gamma^{+}$, we have $x<y$ or $y<x$. If $x<y$, then

$$
\left(P e_{x} \mid e_{y}\right)=\left(P e_{x} \mid T_{y} e_{0}\right)=\left(T_{y}^{*} P e_{x} \mid e_{0}\right)=\left(P T_{y}^{*} e_{x} \mid e_{0}\right)=0
$$


if $x>y$, then $\left(P e_{x} \mid e_{y}\right)=\overline{\left(P e_{y} \mid e_{x}\right)}$ is similarly 0 . So $P e_{x} \perp e_{y}$ for all $y \neq x$, and $P e_{x}$ is a multiple $\lambda_{x} e_{x}$ of $e_{x}$. But

$$
\lambda_{x}=\left(P e_{x} \mid e_{x}\right)=\left(P e_{0} \mid T_{x}^{*} e_{x}\right)=\left(P e_{0} \mid e_{0}\right),
$$

so $\lambda_{x}=\lambda_{0}$ for all $x$. Thus $P=\lambda_{0} 1$, and since $P$ is a projection, $P$ is 0 or 1 .

The map $T: x \mapsto T_{x}$ is a semigroup homomorphism from $\Gamma^{+}$to the semigroup Isom $\mathcal{T}(\Gamma):=\left\{S \in \mathcal{T}(\Gamma): S^{*} S=1\right\}$. A theorem of Murphy ([12, Theorem 2.9], see also [3]), which generalises earlier results of Coburn [7] and Douglas [8], implies that $T: \Gamma^{+} \rightarrow \operatorname{Isom} \mathcal{T}(\Gamma)$ is universal for isometric representations: for every isometric representation $V$ of $\Gamma^{+}$in a $C^{*}$-algebra $B$, there is a homomorphism $\rho_{V}: \mathcal{T}(\Gamma) \rightarrow B$ such that $\rho_{V}\left(T_{x}\right)=V_{x}$ for $x \in \Gamma^{+}$. Murphy's theorem also implies that $\rho_{V}$ is faithful if $V$ satisfies $V_{x} V_{x}^{*} \neq 1$ for $x \neq 0$. From the universal property of $T: \Gamma^{+} \rightarrow \operatorname{Isom} \mathcal{T}(\Gamma)$, it follows that there is a continuous action $\alpha=\alpha^{\Gamma}$ of the compact dual group $\widehat{\Gamma}$ by automorphisms of $\mathcal{T}(\Gamma)$ such that $\alpha_{\gamma}\left(T_{x}\right)=\overline{\gamma(x)} T_{x}$ for $x \in \Gamma^{+}$.

An order ideal in $\Gamma$ is a subgroup $I$ such that $0 \leq x \leq y$ and $y \in I$ imply $x \in I$. Both $I$ and the quotient $\Gamma / I$ are then totally ordered with $I^{+}=I \cap \Gamma^{+}$and $(\Gamma / I)^{+}=\{x+I$ : $\left.x \in \Gamma^{+}\right\}$. Clifford showed in [6] that the group extension $0 \rightarrow I \rightarrow \Gamma \rightarrow \Gamma / I \rightarrow 0$ does not always split. We denote by $\Sigma(\Gamma)$ the set of order ideals in $\Gamma$. Because $\Gamma$ is totally ordered, the set $\Sigma(\Gamma)$ is itself totally ordered by inclusion. As a point of notation, we write $I \subset J$ to include the possibility that $I=J$, and write $I \subsetneq J$ when we mean the inclusion to be strict.

For every order ideal $I$, the map $x \mapsto T_{x+I}^{\Gamma / I}$ is an isometric representation of $\Gamma$, and hence the universal property of $T: \Gamma^{+} \rightarrow \operatorname{Isom} \mathcal{T}(\Gamma)$ gives a homomorphism $Q_{I}: \mathcal{T}(\Gamma) \rightarrow \mathcal{T}(\Gamma / I)$ such that $Q_{I}\left(T_{x}\right)=T_{x+I}^{\Gamma / I}$ for $x \in \Gamma^{+} ; Q_{I}$ is surjective because its range is a $C^{*}$-algebra containing all the generators of $\mathcal{T}(\Gamma / I)$. For $v \in \widehat{\Gamma}$, Lemma 1.1 implies that the composition $Q_{I} \circ \alpha_{v}^{-1}$ is an irreducible representation of $\mathcal{T}(\Gamma)$. In $[\mathbf{4}$, $\S 3]$ we showed that $\operatorname{ker} Q_{I} \circ \alpha_{\nu}^{-1}$ depends only on $\left.v\right|_{I}$, and that every primitive ideal of $\mathcal{T}(\Gamma)$ has this form. So if $X(\Gamma)$ denotes the disjoint union $\bigsqcup\{\widehat{I}: I \in \Sigma(\Gamma)\}$, the map $L: X(\Gamma) \rightarrow \operatorname{Prim} \mathcal{T}(\Gamma)$ defined by

$$
L(I, \gamma)=\operatorname{ker} Q_{I} \circ \alpha_{v}^{-1} \text { where } v \in \widehat{\Gamma} / \text { satisfies }\left.\nu\right|_{I}=\gamma
$$

is a bijection [4, Theorem 3.1]. Our main interest is in determining the topology on $X(\Gamma)$ for which $L$ is a homeomorphism.

2. Adji's structure theorem. Suppose $\Gamma$ is a totally ordered abelian group, and $I$ is an order ideal in $\Gamma$. Our structure theorem describes the relationships between the Toeplitz algebras $\mathcal{T}(I), \mathcal{T}(\Gamma)$, and $\mathcal{T}(\Gamma / I)$.

We have already observed that the universal property of $\mathcal{T}(\Gamma)$ gives a surjection $Q_{I}$ : $\mathcal{T}(\Gamma) \rightarrow \mathcal{T}(\Gamma / I)$ such that $Q_{I}\left(T_{x}\right)=T_{x+I}^{\Gamma / I}$ for $x \in \Gamma^{+}$. Applying the universal property of $\mathcal{T}(I)$ to the restriction of $T^{\Gamma}$ to $I^{+}$gives a homomorphism $\iota_{I}: \mathcal{T}(I) \rightarrow \mathcal{T}(\Gamma)$ such that $\iota_{I}\left(T_{x}^{I}\right)=T_{x}^{\Gamma}$ for $x \in I^{+}$. Since every $T_{x}^{\Gamma}$ is non-unitary, Murphy's theorem implies that $\iota_{I}$ is injective.

The structure theorem in [2] identifies a quotient of $\mathcal{T}(\Gamma)$ as an induced $C^{*}$-algebra. In general, if $G$ is a compact group and $\alpha: H \rightarrow$ Aut $A$ is an action of a closed subgroup $H$ on a $C^{*}$-algebra, the induced $C^{*}$-algebra $\operatorname{Ind}_{H}^{G}(A, \alpha)$ is the subalgebra of $C(G, A)$ consisting of the functions $f$ satisfying $f(g h)=\alpha_{h}^{-1}(f(g))$ for all $g \in G$ and $h \in H$. 
These $C^{*}$-algebras are discussed in $[\mathbf{1 4}, \S 6.3]$. The induced algebra in our theorem is that associated to the dual action $\alpha^{\Gamma / I}$ of $I^{\perp}=(\Gamma / I)^{\wedge}$ on $\mathcal{T}(\Gamma / I)$.

THEOREM 2.1. Let $\Gamma$ be a totally ordered abelian group and I an order ideal in $\Gamma$. Then the ideal $\mathcal{C}(\Gamma, I)$ in $\mathcal{T}(\Gamma)$ generated by $\left\{1-T_{x} T_{x}^{*}: x \in I^{+}\right\}$is Morita equivalent to the commutator ideal $\mathcal{C}(I)$ in $\mathcal{T}(I)$. If we define $\phi_{I}: \mathcal{T}(\Gamma) \rightarrow C(\widehat{\Gamma}, \mathcal{T}(\Gamma / I))$ by

$$
\phi_{I}(a)(\gamma)=Q_{I} \circ \alpha_{\gamma}^{-1}(a)
$$

then $\phi_{I}(a)$ belongs to the induced algebra $\operatorname{Ind}_{I^{\perp}}^{\widehat{\Gamma}}\left(\mathcal{T}(\Gamma / I), \alpha^{\Gamma / I}\right)$, and the following is an exact squence of $C^{*}$-algebras:

$$
0 \rightarrow \mathcal{C}(\Gamma, I) \longrightarrow \mathcal{T}(\Gamma) \stackrel{\phi_{I}}{\longrightarrow} \operatorname{Ind}_{I^{\perp}}^{\widehat{\Gamma}}\left(\mathcal{T}(\Gamma / I), \alpha^{\Gamma / I}\right) \longrightarrow 0 .
$$

This theorem is an improvement of Theorem 3.1 of [2]: the extra ingredient is the Morita equivalence of $\mathcal{C}(\Gamma, I)$ with $\mathcal{C}(I)$. Before proving this extra assertion, we give a more direct proof of [2, Theorem 3.1]; the original proof relied on some general results about semigroup crossed products [1] and a characterisation of induced $C^{*}$-algebras due to Echterhoff [9].

Proof of the exactness in Theorem 2.1. When $\chi \in I^{\perp}=(\Gamma / I)^{\wedge}$, we can check on generators that $Q_{I} \circ \alpha_{\chi}=\alpha_{\chi}^{\Gamma / I} \circ Q_{I}$, and then a straightforward calculation shows that $\phi_{I}(a)$ belongs to $\operatorname{Ind}\left(\mathcal{T}(\Gamma / I), \alpha^{\Gamma / I}\right)$. Further calculations show that $\phi_{I}$ is a homomorphism of $C^{*}$-algebras.

To see that $\phi_{I}$ is surjective, we make two observations. First, we note that, for each fixed $\gamma \in \widehat{\Gamma}$, the set $\left\{\phi_{I}(a)(\gamma): a \in \mathcal{T}(\Gamma)\right\}$ is all of $\mathcal{T}(\Gamma / I)$. Second, we note that for $x \in I^{+}, \phi_{I}\left(T_{x}\right)$ is the function $\varepsilon_{x}: \gamma \rightarrow \gamma(x) 1_{\mathcal{T}(\Gamma / I)}$, which is constant on $I^{\perp}$-orbits; these functions $\left\{\varepsilon_{x}: x \in I^{+}\right\}$generate the $C^{*}$-subalgebra $C\left(\widehat{\Gamma} / I^{\perp}\right) \cong C(\widehat{I})$ of Ind $\left(\mathcal{T}(\Gamma / I), \alpha^{\Gamma / I}\right)$, and hence the range of $\phi_{I}$ contains this $C^{*}$-algebra. Combining these two observations with a partition-of-unity argument on $\widehat{\Gamma} / I^{\perp}$ (as in, for example, the lemma on page 704 of [9]) shows that the range of $\phi_{I}$ is dense in $\operatorname{Ind}\left(\mathcal{T}(\Gamma / I), \alpha^{\Gamma / I}\right)$. Since the range of $\phi_{I}$ is a $C^{*}$-algebra, this implies that $\phi_{I}$ is surjective.

To see that (2.1) is exact we need to prove that $\operatorname{ker} \phi_{I}=\mathcal{C}(\Gamma, I)$. Since $Q_{I}\left(T_{x}\right)=$ $1_{\mathcal{T}(\Gamma / I)}$ for every $x \in I^{+}$, each generator $1-T_{x} T_{x}^{*}$ of $\mathcal{C}(\Gamma, I)$ belongs to ker $\phi_{I}$, and hence $\mathcal{C}(\Gamma, I) \subset \operatorname{ker} \phi_{I}$. Since every ideal is the intersection of the primitive ideals containing it, to prove that $\operatorname{ker} \phi_{I} \subset \mathcal{C}(\Gamma, I)$ it suffices to prove that every irreducible representation $\pi$ of $\mathcal{T}(\Gamma)$ with $\mathcal{C}(\Gamma, I) \subset \operatorname{ker} \pi$ factors through $\phi_{I}$. We know from Proposition 6.16 of [14] that every irreducible representation of $\operatorname{Ind} \mathcal{T}(\Gamma / I)$ has the form $M(\gamma, \rho): f \mapsto \rho(f(\gamma))$ for some $\gamma \in \widehat{\Gamma}$ and some irreducible representation $\rho$ of $\mathcal{T}(\Gamma / I)$. So we suppose that $\pi$ is an irreducible representation of $\mathcal{T}(\Gamma)$ with $\mathcal{C}(\Gamma, I) \subset \operatorname{ker} \pi$, and look for a suitable pair $(\gamma, \rho)$.

Since $\pi\left(1-T_{x} T_{x}^{*}\right)=0$ for $x \in I^{+}$, the map $\pi \circ T$ is an isometric representation of $\Gamma^{+}$which is unitary on $I^{+}$. Since $\Gamma$ is abelian, the operators in the range of $\pi \circ T$ commute; when $x \in I^{+}$, so that $\pi\left(T_{x}\right)$ is a unitary operator, it follows that $\pi\left(T_{x}\right)$ also commutes with every operator of the form $\pi\left(T_{y}\right)^{*}$. Thus the restriction of $\pi \circ T$ to $I^{+}$ has range in $\pi(\mathcal{T}(\Gamma))^{\prime}=\mathbb{C} 1$, and there is a character $\chi$ of $I$ such that $\pi\left(T_{x}\right)=\chi(x) 1$ for all $x \in I^{+}$. We take $\gamma$ to be any character of $\Gamma$ such that $\left.\gamma\right|_{I}=\chi$.

To construct $\rho$, we consider the isometric representation $U: x \mapsto \overline{\gamma(x)} \pi\left(T_{x}\right)$ of $\Gamma^{+}$, which satisfies $U_{x}=1$ for $x \in I^{+}$because $\left.\gamma\right|_{I}=\chi$. We claim that $U$ is also constant on $I$ cosets. To see this, suppose $x, y \in \Gamma^{+}$satisfy $x+I=y+I$. Then $x-y \in I$, and 
since $\Gamma$ is totally ordered, we have either $x-y \geq 0$ or $y-x \geq 0$. If $y-x \geq 0$, then $U_{y}=U_{x+(y-x)}=U_{x} U_{y-x}=U_{x}$, and similarly if $x-y \geq 0$. Thus $U_{x}=U_{y}$, as claimed. We deduce that there is an isometric representation $W$ of $\Gamma / I$ such that $W_{x+I}=U_{x}$, and we take for $\rho$ the representation $\rho_{W}$ of $\mathcal{T}(\Gamma / I)$ such that $\rho_{W}\left(T_{x+I}^{\Gamma / I}\right)=W_{x+I}$.

To check that $\pi=M(\gamma, \rho) \circ \phi_{I}$, we let $x \in \Gamma^{+}$and compute:

$$
M(\gamma, \rho) \circ \phi_{I}\left(T_{x}\right)=\rho\left(Q_{I}\left(\left(\alpha_{\gamma}\right)^{-1}\left(T_{x}\right)\right)\right)=\gamma(x) \rho\left(T_{x+I}^{\Gamma / I}\right)=\gamma(x) U_{x}=\pi\left(T_{x}\right) .
$$

Thus $\pi$ factors through $\phi_{I}$, as required, and we have proved exactness of (2.1).

We still have to prove the Morita equivalence of $\mathcal{C}(\Gamma, I)$ and $\mathcal{C}(I)$. For this we use the following general lemma which is surely part of the folklore. We adopt the convention that if $Y$ and $Z$ are subspaces of a $C^{*}$-algebra, then $Y Z$ denotes the linear span of the products $\{y z: y \in Y, z \in Z\}$.

Lemma 2.2. Suppose that $D$ is a $C^{*}$-algebra, $E$ is a $C^{*}$-subalgebra of $D$, and $X$ is a closed vector subspace of $D$ which contains $E$ and satisfies

$$
E X \subset X, X D \subset X, X X^{*} \subset E \text {, and } X^{*} X \text { is dense in } D \text {. }
$$

Then $X$ is an $E-D$ imprimitivity bimodule with

$$
e \cdot x:=e x, x \cdot d:=x d,{ }_{E}\langle x, y\rangle:=x y^{*}, \text { and }\langle x, y\rangle_{D}:=x^{*} y .
$$

The Rieffel correspondence associated to this bimodule takes an ideal $J$ in $D$ to the ideal $J \cap E$ in $E$.

Proof. It follows from the hypotheses and the usual algebraic properties of a $C^{*}$ algebra that $X$ is an $E-D$ bimodule, that the given formulas define $C^{*}$-algebra-valued inner products on $X$, and that the two inner products are compatible in the required sense (that is, they satisfy properties (b) and (c) of [14, Definition 3.1]). Since

$$
\|x\|_{D}^{2}=\left\|\langle x, x\rangle_{D}\right\|=\left\|x^{*} x\right\|=\|x\|^{2},
$$

the norm induced by these inner products coincides with the norm coming from $D$, and $X$ is complete in this norm. Since every $C^{*}$-algebra satisfies $E E^{*}=E$ and $E \subset X$, $X$ is full as a left Hilbert $E$-module; the density of $X^{*} X$ in $D$ implies that it is full as a right-Hilbert $D$-module. Thus $E$ is an $E-D$ imprimitivity bimodule with the operations (2.3).

If $J$ is an ideal in $D$, the corresponding ideal $X$-Ind $J$ in $E$ is by definition

$$
X \text {-Ind } J=\overline{\operatorname{span}}\left\{{ }_{E}\langle x j, y\rangle: x, y \in X, j \in J\right\}=\overline{\operatorname{span}}\left\{x j y^{*}: x, y \in X, j \in J\right\}
$$

(see $[14, \S 3.3])$. This is clearly contained in $J \cap E$. On the other hand, if $j \in J \cap E$, then we can approximate $j$ by elements of the form $b j c^{*}$ with $b, c \in E$, and $b j c^{*}$ belongs to $X$-Ind $J$ because $E \subset X$.

We aim to apply Lemma 2.2 with $D=\mathcal{C}(\Gamma, I)$ and $E=\iota_{I}(\mathcal{C}(I))$. The description of $\mathcal{C}(\Gamma, I)$ in the next lemma will suggest a natural candidate for $X$, and will also allow us to verify the properties (2.2) by direct calculations. To simplify the formulas in these calculations, we write $1_{x}$ for $T_{x} T_{x}^{*}$, and recall that $\left\{1_{x}: x \in \Gamma^{+}\right\}$is a commuting family of projections satisfying $1_{x} 1_{y}=1_{\max \{x, y\}}$. The key ingredient in the calculations is the 
following formula for $a, u \in \Gamma^{+}$:

$$
T_{a}^{*}\left(1-1_{u}\right)= \begin{cases}\left(1-1_{u-a}\right) T_{a}^{*} & \text { if } a<u \\ 0 & \text { if } u \leq a\end{cases}
$$

LeMmA 2.3. The ideal $\mathcal{C}(\Gamma, I)$ in $\mathcal{T}(\Gamma)$ generated by $\left\{1-1_{u}: u \in I^{+}\right\}$is given by

$$
\mathcal{C}(\Gamma, I)=\overline{\operatorname{span}}\left\{T_{x}\left(1-1_{u}\right) T_{y}^{*}: x, y \in \Gamma^{+}, u \in I^{+}\right\}
$$

Proof. Since the right-hand side of (2.5) contains all the generators of $\mathcal{C}(\Gamma, I)$ and is certainly contained in $\mathcal{C}(\Gamma, I)$, it suffices to show that the right-hand side of $(2.5)$ is an ideal in $\mathcal{T}(\Gamma)$. Since it is closed under taking adjoints and left multiplication by $T_{z}$, it suffices to prove that it is closed under left multiplication by $T_{z}^{*}$. So we let $z \in \Gamma^{+}$, let $T_{x}\left(1-1_{u}\right) T_{y}^{*}$ be a typical spanning member of the right-hand side, and compute using (2.4):

$$
\begin{aligned}
T_{z}^{*}\left(T_{x}\left(1-1_{u}\right) T_{y}^{*}\right) & = \begin{cases}T_{x-z}\left(1-1_{u}\right) T_{y}^{*} & \text { if } z<x \\
T_{z-x}^{*}\left(1-1_{u}\right) T_{y}^{*} & \text { if } x \leq z .\end{cases} \\
& = \begin{cases}T_{x-z}\left(1-1_{u}\right) T_{y}^{*} & \text { if } z<x \\
\left(1-1_{u+x-z}\right) T_{y+z-x}^{*} & \text { if } x \leq z<x+u \\
0 & \text { if } x+u \leq z .\end{cases}
\end{aligned}
$$

In the middle case we have $0 \leq u+x-z=u-(z-x) \leq u$, which because $I$ is an order ideal implies $u+x-z \in I$; thus (2.6) always belongs to the right-hand side of (2.5).

Proof of the Morita equivalence in Theorem 2.1. As we mentioned above, we intend to apply Lemma 2.2 with $D=\mathcal{C}(\Gamma, I)$ and $E=\iota_{I}(\mathcal{C}(I))$. We take for $X$ the closed subspace

$$
X:=\overline{\operatorname{span}}\left\{T_{v}\left(1-1_{u}\right) T_{y}^{*}: y \in \Gamma^{+}, u, v \in I^{+}\right\}
$$

of $D=\mathcal{C}(\Gamma, I) \subset \mathcal{T}(\Gamma)$, and notice that Lemma 2.3 immediately implies that $X^{*} X$ is dense in $E$. Applying Lemma 2.3 with $\Gamma=I$ shows that the image $E=\iota_{I}(\mathcal{C}(I))$ of the commutator ideal $\mathcal{C}(I)$ in $\mathcal{T}(I)$ is

$$
E=\overline{\operatorname{span}}\left\{T_{v}\left(1-1_{u}\right) T_{t}^{*}: u, v, t \in I^{+}\right\},
$$

so we certainly have $E \subset X$.

To see that $X$ has the other three properties we require, we let $u, v \in I^{+}, w, x, y, z \in$ $\Gamma^{+}$, and premultiply $(2.6)$ by $T_{w}\left(1-1_{v}\right)$ to get

$$
\left(T_{w}\left(1-1_{v}\right) T_{z}^{*}\right)\left(T_{x}\left(1-1_{u}\right) T_{y}^{*}\right)= \begin{cases}T_{w}\left(1-1_{v}\right) T_{x-z}\left(1-1_{u}\right) T_{y}^{*} & \text { if } z<x \\ T_{w}\left(1-1_{v}\right)\left(1-1_{u+x-z}\right) T_{y+z-x}^{*} & \text { if } x \leq z<x+u \\ 0 & \text { if } x+u \leq z\end{cases}
$$


In the first case, we can now use the adjoint of (2.4) to further simplify, obtaining

$$
\left(T_{w}\left(1-1_{v}\right) T_{z}^{*}\right)\left(T_{x}\left(1-1_{u}\right) T_{y}^{*}\right)= \begin{cases}0 & \text { if } z \leq x-v \\ T_{w+x-z}\left(1-1_{v-(x-z)}\right)\left(1-1_{u}\right) T_{y}^{*} & \text { if } x-v<z<x \\ T_{w}\left(1-1_{v}\right)\left(1-1_{u+x-z}\right) T_{y+z-x}^{*} & \text { if } x \leq z<x+u \\ 0 & \text { if } x+u \leq z\end{cases}
$$

To see that $E X \subset X$, we just note that if $w, x$ and $z$ are all in $I^{+}$, then $w+x-z$ is in $I^{+}$ too, and both formulas for the product in (2.8) lie in $X$. To see that $X D \subset X$, we note that the formulas in (2.8) vanish unless $x-v<z<x+u$, in which case $z-x$ lies in $I$. To check that $X X^{*}$ is contained in $E$, we suppose that $w$ and $y$ lie in $I^{+}$. Then in the second case in (2.8) we have

$$
0 \leq w+x-z \leq w+v \in I^{+} \text {and } 0 \leq v-(x-z) \leq v \in I^{+},
$$

both $w+x-z$ and $v-(x-z)$ belong to $I^{+}$, and the formula defines an element of $E$. A similar argument applies to the third case in (2.8).

We have now verified that $E$ satisfies the hypotheses of Lemma 2.2, and hence the Morita equivalence of $\mathcal{C}(I) \cong E$ and $D=\mathcal{C}(\Gamma, I)$ follows from that lemma. This completes the proof of Theorem 2.1.

REMARK 2.4. In the proof of Theorem 2.1, we wrote down a specific bimodule (2.7) which implements the equivalence. Since this bimodule is one of the kind described in Lemma 2.2, we can deduce from Lemma 2.2 that the Rieffel correspondence associated to the bimodule takes an ideal $J$ in $\mathcal{C}(\Gamma, I)$ to the ideal $\iota_{I}^{-1}\left(J \cap \iota_{I}(\mathcal{C}(I))\right)$ in $\mathcal{C}(I)$.

3. The main theorem. A totally ordered set $\Sigma$ is said to be well-ordered if every subset of $\Sigma$ has a least element, or equivalently if every element of $\Sigma$ has a successor. Our main theorem asserts that the topology on $\operatorname{Prim} \mathcal{T}(\Gamma)$ is given by the upwardslooking topology on $\bigsqcup \widehat{I}$, as defined in the introduction, if and only if the set $\Sigma(\Gamma)$ of order ideals in $\Gamma$ is well-ordered by inclusion.

THEOREM 3.1. Let $\Gamma$ be a totally ordered abelian group, and denote by $X(\Gamma)$ the disjoint union $\bigsqcup\{\widehat{I}: I \in \Sigma(\Gamma)\}$. The map $L: X(\Gamma) \rightarrow \operatorname{Prim} \mathcal{T}(\Gamma)$ is a homeomorphism for the upwards-looking topology on $X(\Gamma)$ if and only if $\Sigma(\Gamma)$ is well-ordered.

To prepare for the proof of Theorem 3.1 , we recall from $[4, \S 4]$ some general results relating the upwards-looking topology to the topology on Prim $\mathcal{T}(\Gamma)$. In the last part, when $I$ is an order ideal in $\Gamma$, we view $X(I)=\bigsqcup\{\widehat{J}: J \in \Sigma(\Gamma), J \subset I\}$ as a subset of $X(\Gamma)$. Notice that the last part of the Proposition applies to every order ideal $I$ when $\Sigma(\Gamma)$ is well-ordered.

Proposition 3.2. Let $\Gamma$ be a totally ordered abelian group, let $F$ be a subset of $X(\Gamma)$, and let $\bar{F}$ denote the upwards-looking closure of $F$. Then

(a) $L(\bar{F}) \subset \overline{L(F)}$;

(b) if $F$ is contained in a single $\widehat{I}$, then $L(\bar{F})=\overline{L(F)}$;

(c) if $I$ is an order ideal which has a successor in $\Sigma(\Gamma)$ and $L(I, \gamma) \in \overline{L(F)}$, then $L(I, \gamma) \in \overline{L(F \cap X(I))}$.

Proof. These three assertions are proved in, respectively, Lemma 4.3, Proposition 4.4 and Lemma 4.9 of [4]. 
To these general properties of the upwards-looking topology, we add the following.

Proposition 3.3. Suppose that I is an order ideal in a totally ordered abelian group $\Gamma$, and $F$ is a subset of $X(\Gamma)$ which lies entirely in $X(I)$. Suppose that $\chi \in \widehat{I}$ and $L(I, \chi)$ belongs to the closure $\overline{L(F)}$ of $L(F)$ in $\operatorname{Prim} \mathcal{T}(\Gamma)$. Then $(I, \chi)$ belongs to the closure of $F$ in the upwards-looking topology on $X(\Gamma)$.

This proposition is our major innovation, and its proof uses classical Toeplitz operators, as in $[12, \S 3]$, for example. So we review some basic properties of these operators. Suppose $\Gamma$ is a totally ordered abelian group with positive cone $\Gamma^{+}$, and $\widehat{\Gamma}$ is the dual of $\Gamma$. Then $L^{2}(\widehat{\Gamma})$ has a natural orthonormal basis $\left\{\varepsilon_{x}: x \in \Gamma\right\}$ consisting of the evaluation functions $\varepsilon_{x}: \gamma \rightarrow \gamma(x)$. The Hardy space of $\Gamma$ is the closed span $H^{2}(\widehat{\Gamma}):=\overline{\operatorname{span}}\left\{\varepsilon_{x}: x \in \Gamma^{+}\right\}$, and we denote by $P$ the orthogonal projection of $L^{2}(\widehat{\Gamma})$ on $H^{2}(\widehat{\Gamma})$. For $f \in C(\widehat{\Gamma})$, the Toeplitz operator with symbol $f$ is the bounded operator $T_{f}$ on $H^{2}(\widehat{\Gamma})$ defined by $T_{f} h:=P(f h)$, where $f h$ is the usual pointwise product of $f$ and $h$. The map $f \mapsto T_{f}$ of $C(\widehat{\Gamma})$ into $B\left(H^{2}(\widehat{\Gamma})\right)$ is norm-decreasing and $*$-linear, but is not multiplicative. Thus a Toeplitz operator $T_{f}$ with invertible symbol $f$ is not in general invertible. Indeed, Toeplitz operators have traditionally been of interest because the Toeplitz operators with invertible symbol are Fredholm operators in some generalised sense, and have an interesting index theory (see [5], for example).

We want to view the Toeplitz operators $T_{f}$ as elements of the Toeplitz algebra $\mathcal{T}(\Gamma)$. To do this, we use the unitary isomorphism $\mathcal{F}: \ell^{2}(\Gamma) \rightarrow L^{2}(\widehat{\Gamma})$ which extends the Fourier transform

$$
(\mathcal{F} f)(\gamma):=\sum_{x \in \Gamma} f(x) \gamma(x)
$$

The unitary $\mathcal{F}$ maps the orthonormal basis $\left\{e_{x}: x \in \Gamma\right\}$ into $\left\{\varepsilon_{x}: x \in \Gamma\right\}$, and hence restricts to a unitary isomorphism of $\ell^{2}\left(\Gamma^{+}\right)$onto $H^{2}(\widehat{\Gamma})$, which we still denote by $\mathcal{F}$. One quickly checks that $\mathcal{F} T_{x} \mathcal{F}^{*}=T_{\varepsilon_{x}}$ for $x \in \Gamma^{+}$, and since $\operatorname{Ad} \mathcal{F}: T \mapsto \mathcal{F} T \mathcal{F}^{*}$ is a *-isomorphism and $T_{f}^{*}=T_{f^{*}}$, we also have

$$
\mathcal{F} T_{x}^{*} \mathcal{F}^{*}=T_{\varepsilon_{x}}^{*}=T_{\varepsilon_{x}^{*}}=T_{\varepsilon_{-x}} .
$$

Thus for every trigonometric polynomial $p=\sum_{y \in \Gamma} \lambda_{y} \varepsilon_{y}$, the Toeplitz operator $T_{p}=$ $\sum_{y \in \Gamma} \lambda_{y} T_{\varepsilon_{y}}$ belongs to $\operatorname{Ad} \mathcal{F}(\mathcal{T}(\Gamma))$. Since the trigonometric polynomials are dense in $C(\widehat{\Gamma})$ and $f \mapsto T_{f}$ is norm-decreasing, this implies that $\operatorname{Ad} \mathcal{F}$ is an isomorphism of $\mathcal{T}(\Gamma)$ onto the $C^{*}$-algebra generated by the Toeplitz operators $\left\{T_{f}: f \in C(\widehat{\Gamma})\right\}$.

We shall use the inverse isomorphism Ad $\mathcal{F}^{*}$ to view the Toeplitz operators $T_{f}$ as elements of $\mathcal{T}(\Gamma)$. Notice that, with this identification, Equation (3.1) implies that for a trigonometric polynomial $p=\sum_{y \in \Gamma} \lambda_{y} \varepsilon_{y}$ we have

$$
T_{p}=\sum_{y \geq 0} \lambda_{y} T_{y}+\sum_{y<0} \lambda_{y} T_{-y}^{*} .
$$

One standard property of Toeplitz operators which we will need says that $T_{f}=0$ implies $f=0$. In fact this follows quite easily from Theorem 2.1. To see this, note that when $I=\Gamma$, the Toeplitz algebra $\mathcal{T}(\Gamma / \Gamma)$ is just $\mathbb{C} 1$, and $\operatorname{Ind}_{\Gamma^{\perp}} \mathcal{T}(\Gamma / \Gamma)$ is naturally identified with $C(\widehat{\Gamma})$. A straightforward computation using (3.2) shows that the homomorphism $\phi_{\Gamma}$ of Theorem 2.1 satisfies $\phi_{\Gamma}\left(T_{p}\right)(\gamma)=p(\gamma)$ for every trigonometric polynomial $p$, and the resulting equation $\phi_{\Gamma}\left(T_{p}\right)=p$ extends by continuity to all 
$p \in C(\widehat{\Gamma})$. Since both $f \mapsto T_{f}$ and $\phi_{\Gamma}$ are norm-decreasing, this implies that $\left\|T_{f}\right\|=$ $\|f\|_{\infty}$, and in particular we can deduce that $T_{f}=0$ implies $f=0$, as claimed.

We now need to know how the Toeplitz operators interact with the other ingredients in Theorem 2.1.

Proposition 3.4. Suppose $f \in C(\widehat{\Gamma})$, and view $T_{f}$ as an element of $\mathcal{T}(\Gamma)$.

(a) For $\gamma \in \widehat{\Gamma}, \alpha_{\gamma}\left(T_{f}\right)$ is the Toeplitz operator $T_{\mathrm{lt}_{\gamma}(f)}$ whose symbol is the left translate off by $\gamma: \mathrm{lt}_{\gamma}(f)(v)=f\left(\gamma^{-1} v\right)$.

(b) If $J$ is an order ideal in $\Gamma$, then $Q_{J}\left(T_{f}\right)$ is the Toeplitz operator $T_{\left.f\right|_{J \perp} / J}$ whose symbol is the function on $(\Gamma / J)^{\wedge}$ obtained by identifying $(\Gamma / J)^{\wedge}$ with the subset $J^{\perp}$ of $\widehat{\Gamma}$ and restricting $f$ to $J^{\perp}$.

Proof. If $p=\sum_{y \in \Gamma} \lambda_{y} \varepsilon_{y}$ is a trigonometric polynomial, then a computation using (3.2) shows that $\alpha_{\gamma}\left(T_{p}\right)=T_{q}$, where $q=\sum_{y \in \Gamma} \lambda_{y} \overline{\gamma(x)} \varepsilon_{y}$, and another computation shows that $q=1 \mathrm{t}_{\gamma}(p)$. This gives (a) for trigonometric polynomials, and the general statement follows because both $\alpha_{\gamma}\left(T_{f}\right)$ and $T_{\mathrm{lt}_{\gamma}(f)}$ are continuous in $f$.

For (b), we note that the identification of $J^{\perp}$ with $(\Gamma / J)^{\wedge}$ carries $\left.\varepsilon_{x}\right|_{J^{\perp}}$ into $\varepsilon_{x+J}$, and hence carries the restriction $\left.p\right|_{J^{\perp}}$ of $p=\sum_{y \in \Gamma} \lambda_{y} \varepsilon_{y}$ into $\sum_{y \in \Gamma} \lambda_{y} \varepsilon_{y+J}$. Now another application of (3.2) shows that $Q_{J}\left(T_{p}\right)=T_{\left.p\right|_{J \perp} \perp}^{\Gamma / J}$, and this extends to arbitrary functions in $C(\widehat{\Gamma})$ by continuity.

Proof of Proposition 3.3. We suppose that $\chi$ does not belong to the closure of $F$, and produce an element $T$ of

$$
k(L(F)):=\bigcap\{L(J, \lambda):(J, \lambda) \in F\}
$$

which does not belong to $L(I, \chi)$. To define $T$, we consider the restriction map Res : $\left.v \mapsto v\right|_{I}$ of $\widehat{\Gamma}$ onto $\widehat{I}$. We choose $v \in \widehat{\Gamma}$ such that $\left.v\right|_{I}=\chi$. Since $\bar{F}$ is closed, so is $\operatorname{Res}^{-1}(\bar{F})$, and we can find a function $f \in C(\widehat{\Gamma})$ such that $f(v)=1$ and $f$ vanishes on $\operatorname{Res}^{-1}(\bar{F})$. We claim that $T=T_{f}$ has the required properties.

We first show that $T_{f} \notin L(I, \chi)$. Proposition 3.4 implies that

$$
Q_{I}\left(\alpha_{v}^{-1}\left(T_{f}\right)\right)=Q_{I}\left(T_{1 t_{v}^{-1}(f)}\right)=T_{\left.\mathrm{lt}_{v}^{-1}(f)\right|_{I^{\perp}}}^{\Gamma / I} ;
$$

since $\mathrm{lt}_{v}^{-1}(f)(1)=f(v)=1, g:=\left.\mathrm{lt}_{v}^{-1}(f)\right|_{I^{\perp}}$ is a non-zero element of $C\left((\Gamma / I)^{\wedge}\right)$, and the Toeplitz operator $T_{g}^{\Gamma / I}$ is non-zero. Hence (3.4) implies that $T_{f} \notin L(I, \chi)$. On the other hand, suppose $(J, \lambda) \in F$ and $\gamma \in \widehat{\Gamma}$ satisfies $\left.\gamma\right|_{J}=\lambda$. The hypothesis on $F$ says that $J \subset I$, and thus $\operatorname{Res}\left(\gamma J^{\perp}\right) \subset \bar{F}$. Thus $f$ vanishes on $\gamma J^{\perp}$,

$$
Q_{J}\left(\alpha_{\gamma}^{-1}\left(T_{f}\right)\right)=T_{\left.\mathrm{lt}_{\gamma}^{-1}(f)\right|_{J \perp}}^{\Gamma / J}=0,
$$

and $T_{f} \in L(J, \lambda)$. So $T_{f}$ belongs to the intersection $k(L(F))$ in (3.3), and $T=T_{f}$ has both required properties.

Proof of Theorem 3.1. We first suppose that $\Sigma(\Gamma)$ is well-ordered. Let $F$ be a subset of $X(\Gamma)$, and suppose that $L(I, \chi)$ belongs to the closure of $L(F)$ in $\operatorname{Prim} \mathcal{T}(\Gamma)$. Since $\Sigma(\Gamma)$ is well-ordered, either $I=\Gamma$ or $I$ has a successor in $\Sigma(\Gamma)$. If $I=\Gamma$, then Proposition 3.3 implies that $(I, \chi)$ belongs to the closure of $F$. If $I$ has a successor, then part (c) of Proposition 3.2 implies that $L(I, \chi)$ belongs to the closure of $L(F \cap X(I))$, 
and Proposition 3.3 implies that $(I, \chi) \in \bar{F}$. Thus

$$
L(I, \chi) \in \overline{L(F)} \Longrightarrow(I, \chi) \in \bar{F} \Longrightarrow L(I, \chi) \in L(\bar{F}),
$$

which by part (a) of Proposition 3.2 implies that $\overline{L(F)}=L(\bar{F})$, and $L$ is a homeomorphism.

For the other direction, suppose that $\Sigma(\Gamma)$ is not well-ordered, so that there is a subset $S$ of $\Sigma(\Gamma)$ which does not have a least element. Let $I=\bigcap_{J \in S} J$. Then $I$ is an order ideal in $\Gamma$ which is the greatest lower bound for $S$ in $\Sigma(\Gamma)$, and hence cannot belong to $S$. Let $\phi_{I}: \mathcal{T}(\Gamma) \rightarrow \operatorname{Ind}_{I^{\perp}}^{\widehat{\Gamma}} \mathcal{T}(\Gamma / I)$ be the homomorphism of Theorem 2.1. We claim that $\operatorname{ker} \phi_{I}=\bigcap_{J \in S} \operatorname{ker} \phi_{J}$.

To prove the claim, we first consider the quotient maps $Q_{J / I}: \mathcal{T}(\Gamma / I) \rightarrow \mathcal{T}(\Gamma / J)$, and observe that $\bigoplus_{J \in S} Q_{J / I}(x+I)$ is unitary if and only if $x+I \in J / I$ for all $J \in S$, and hence if and only if $x \in I=\bigcap_{J \in S} J$. Thus Murphy's theorem implies that $\bigoplus_{J \in S} Q_{J / I}$ is faithful on $\mathcal{T}(\Gamma / I)$, and we have

$$
\operatorname{ker} Q_{I}=\operatorname{ker}\left(\left(\bigoplus_{J \in S} Q_{J / I}\right) \circ Q_{I}\right)=\operatorname{ker}\left(\bigoplus_{J \in S} Q_{J}\right)=\bigcap_{J \in S} \operatorname{ker} Q_{J} .
$$

Now

$$
\begin{aligned}
\operatorname{ker} \phi_{I} & =\left\{a \in \mathcal{T}(\Gamma): \alpha_{\gamma}^{-1}(a) \in \operatorname{ker} Q_{I} \text { for all } \gamma \in \widehat{\Gamma}\right\} \\
& =\left\{a \in \mathcal{T}(\Gamma): \alpha_{\gamma}^{-1}(a) \in \bigcap_{J \in S} \operatorname{ker} Q_{J} \text { for all } \gamma \in \widehat{\Gamma}\right\} \\
& =\bigcap_{J \in S}\left\{a \in \mathcal{T}(\Gamma): \alpha_{\gamma}^{-1}(a) \in \operatorname{ker} Q_{J} \text { for all } \gamma \in \widehat{\Gamma}\right\} \\
& =\bigcap_{J \in S} \operatorname{ker} \phi_{J},
\end{aligned}
$$

as claimed.

Since $\operatorname{ker} \phi_{I}=\bigcap_{\gamma \in \widehat{I}} L(I, \gamma)$, the claim implies that $\{L(I, \gamma): \gamma \in \widehat{I}\}$ is contained in the closure of $\{L(J, \chi): J \in S, \chi \in \widehat{J}\}$ in Prim $\mathcal{T}(\Gamma)$. But because $I$ does not contain any element of $S,\{(I, \gamma): \gamma \in \widehat{I}\}$ is not contained in the closure of $\{(J, \chi): J \in S\}$ in the upwards-looking topology. Therefore $L$ is not a homeomorphism for this topology.

4. Group extensions. In this section, we prove the following theorem.

THEOREM 4.1. Let $\Gamma$ be a totally ordered abelian group and I an order ideal in $\Gamma$.

(a) Suppose that $\Sigma(I)$ is well-ordered, and give the set

$$
X_{0}(I):=\bigsqcup\{\widehat{J}: J \in \Sigma(I), J \neq I\}
$$

the upwards-looking topology. Then $L: X_{0}(I) \rightarrow \operatorname{Prim} \mathcal{T}(\Gamma)$ is a homeomorphism of $X_{0}(I)$ onto the open subset $\mathcal{O}_{\mathcal{C}(\Gamma, I)}=\{P \in \operatorname{Prim} \mathcal{T}(\Gamma): \mathcal{C}(\Gamma, I) \not \subset P\}$.

(b) Suppose that $\Sigma(\Gamma / I)$ is well-ordered, and give the set

$$
X(\Gamma, I):=\bigsqcup\{\widehat{J}: J \in \Sigma(\Gamma), I \subset J\}
$$


the upwards-looking topology. Then $L: X(\Gamma, I) \rightarrow \operatorname{Prim} \mathcal{T}(\Gamma)$ is a homeomorphism of $X(\Gamma, I)$ onto the closed subset $\{P \in \operatorname{Prim} \mathcal{T}(\Gamma): \mathcal{C}(\Gamma, I) \subset P\}$.

The assertions about $\mathcal{O}_{\mathcal{C}(\Gamma, I)}$ will follow from Theorem 2.1. Much of the argument applies to arbitrary order ideals $I$ :

Proposition 4.2. Suppose I is an order ideal in a totally ordered abelian group $\Gamma$. Then

$$
\mathcal{O}_{\mathcal{C}(\Gamma, I)}:=\{P \in \operatorname{Prim} \mathcal{T}(\Gamma): \mathcal{C}(\Gamma, I) \not \subset P\} \text { is }\{L(J, \gamma): J \subsetneq I, J \in \Sigma(\Gamma)\} .
$$

For $J \in \Sigma(\Gamma)$ satisfying $J \subsetneq I$ and $\gamma \in \widehat{J}$, we have

$$
L^{I}(J, \gamma)=\iota_{I}^{-1}\left(L(J, \gamma) \cap \iota_{I}(\mathcal{T}(I))\right) .
$$

The map $L(J, \gamma) \mapsto L^{I}(J, \gamma)$ is a homeomorphism of $\mathcal{O}_{\mathcal{C}(\Gamma, I)}$ onto the subset $\mathcal{O}_{\mathcal{C}(I)}$ of $\mathcal{T}(I)$ which is naturally homeomorphic to $\operatorname{Prim} \mathcal{C}(I)$.

Proof. For the assertion (4.1), it suffices to show that

$$
\mathcal{C}(\Gamma, I) \subset L(J, \gamma) \text { if and only if } I \subset J .
$$

If $I \subset J$, then $\mathcal{C}(\Gamma, I) \subset \mathcal{C}(\Gamma, J)$, and the exactness in Theorem 2.1 implies that

$$
\mathcal{C}(\Gamma, I) \subset \mathcal{C}(\Gamma, J)=\operatorname{ker} \phi_{J}=\bigcap_{\nu \in \widehat{\Gamma}} L\left(J,\left.\nu\right|_{J}\right) \subset L(J, \gamma) .
$$

On the other hand, suppose $\mathcal{C}(\Gamma, I) \subset L(J, \gamma)$, choose $v \in \widehat{\Gamma}$ satisfying $\left.\nu\right|_{J}=\gamma$, and let $x \in I^{+}$. Then $1-T_{x} T_{x}^{*} \in \mathcal{C}(\Gamma, I) \subset L(J, \gamma)$, so

$$
1-T_{x+J}^{\Gamma / J}\left(T_{x+J}^{\Gamma / J}\right)^{*}=Q_{J} \circ \alpha_{v}^{-1}\left(1-T_{x} T_{x}^{*}\right)=0 .
$$

Thus $T_{x+J}^{\Gamma / J}$ is unitary, and $x \in J$. This implies that $I=I^{+}-I^{+} \subset J$, as required, and we have proved (4.1).

To prove (4.2), we consider the map $Q_{J}^{I}$ of $\mathcal{T}(I)$ onto $\mathcal{T}(I / J)$ characterised by $Q_{J}^{I}\left(T_{x}^{I}\right)=T_{x+J}^{I / J}$. We can verify on generators that $Q_{J} \circ \iota_{I}=\iota_{I / J} \circ Q_{J}^{I}$. We now choose $\chi \in \widehat{\Gamma}$ such that $\left.\chi\right|_{J}=\gamma$, and write $v=\left.\chi\right|_{I}$. Then

$$
\begin{aligned}
L^{I}(J, \gamma) & =\left\{\xi \in \mathcal{T}(I): Q_{J}^{I} \circ\left(\alpha_{\nu}^{I}\right)^{-1}(\xi)=0\right\} \\
& =\left\{\xi \in \mathcal{T}(I): \iota_{I / J} \circ Q_{J}^{I} \circ\left(\alpha_{\nu}^{I}\right)^{-1}(\xi)=0\right\} \\
& =\left\{\xi \in \mathcal{T}(I): Q_{J} \circ \iota_{I} \circ\left(\alpha_{\nu}^{I}\right)^{-1}(\xi)=0\right\} \\
& =\left\{\xi \in \mathcal{T}(I): Q_{J} \circ \alpha_{\chi}^{-1} \circ \iota_{I}(\xi)=0\right\} \\
& =\left\{\xi \in \mathcal{T}(I): \iota_{I}(\xi) \in L(J, \gamma)\right\},
\end{aligned}
$$

which is (4.2).

Since the natural homeomorphism of $\mathcal{O}_{\mathcal{C}(\Gamma, I)}$ onto $\operatorname{Prim} \mathcal{C}(\Gamma, I)$ takes a primitive ideal $P$ to $P \cap \mathcal{C}(\Gamma, I)$, composing this homeomorphism with the homeomorphism onto $\operatorname{Prim} \mathcal{C}(I)$ described in Remark 2.4 gives a homeomorphism of $\mathcal{O}_{\mathcal{C}(\Gamma, I)}$ onto $\operatorname{Prim} \mathcal{C}(I)$ which takes $P$ to $\iota_{I}^{-1}\left(P \cap \iota_{I}(\mathcal{C}(I))\right)$. Equation (4.2) implies that this homeomorphism takes $L(J, \gamma)$ to $L^{I}(J, \gamma) \cap \mathcal{C}(I)$. 
Proof of Theorem 4.1. Suppose that $\Sigma(I)$ is well-ordered. Then we know from Theorem 3.1 that the map $L^{I}$ is a homeomorphism of $X(I)=X_{0}(I) \sqcup \widehat{I}$ onto $\operatorname{Prim} \mathcal{T}(I)$. The last comment in Proposition 4.2 says that the range of this map is $\mathcal{O}_{\mathcal{C}(I)}$, and the composition of $L^{I}$ with the homeomorphism of $\mathcal{O}_{\mathcal{C}(I)}$ onto $\mathcal{O}_{\mathcal{C}(\Gamma, I)}$ is $L: X_{0}(I) \rightarrow$ $\mathcal{O}_{\mathcal{C}(\Gamma, I)}$. This gives part (a).

For part (b), suppose $\Sigma(\Gamma / I)$ is well-ordered and $F$ is a subset of $X(\Gamma, I)$, and $L(J, \chi) \in \overline{L(F)}$; we want to prove that $(J, \chi) \in \bar{F}$. However, the hypotheses on $\Sigma(\Gamma / I)$ means that either $J=\Gamma$ or $J$ has a successor, and the argument of the first paragraph of the proof of Theorem 3.1 (with $I$ replaced by $J$ ) shows that $(J, \chi) \in \bar{F}$.

REMARK 4.3. One would have liked to use this result to study $\operatorname{Prim} \mathcal{T}(\Gamma)$ when $\Sigma(\Gamma)$ is not well-ordered, by using a composition series for $\Gamma$ in which the subquotients have $\Sigma$ well-ordered. If $\Sigma(\Gamma)$ contains an order ideal $I$ which does not have a successor, then part (a) of Theorem 4.1 gives useful information. However, in the quotient $\Gamma / I$, the trivial ideal $I / I$ still does not have a successor, so $\Sigma(\Gamma / I)$ is not well-ordered, and part (b) does not apply. When there is just one such ideal $I$, just the trivial ideal in $\Gamma / I$ will lack a successor, and we can hope to describe the topology on Prim $\mathcal{T}(\Gamma / I)$ by adjusting the upwards-looking topology as in [4, Theorem 4.13]. In general, though, the structure of $\Sigma(\Gamma)$ could be much more complicated than this.

\section{REFERENCES}

1. S. Adji, Invariant ideals of crossed products by semigroups of endomorphisms, in Functional analysis and global analysis (T. Sunada and P. W. Sy, Eds.) (Springer-Verlag, 1997), $1-8$.

2. S. Adji, Semigroup crossed products and the structure of Toeplitz algebras, J. Operator Theory 44 (2000), 139-150.

3. S. Adji, M. Laca, M. Nilsen and I. Raeburn, Crossed products by semigroups of endomorphisms and the Toeplitz algebras of ordered groups, Proc. Amer. Math. Soc. 122 (1994), $1133-1141$.

4. S. Adji and I. Raeburn, The ideal structure of Toeplitz algebras, Integral Equations and Operator Theory 48 (2004), 281-293.

5. S. Adji, I. Raeburn and A. Ströh, An index theorem for Toeplitz algebras on totally ordered groups, Proc. Amer. Math. Soc. 126 (1998), 2993-2998.

6. A. H. Clifford, Note on Hahn's theorem on ordered groups, Proc. Amer. Math. Soc. 5 (1954), 860-863.

7. L. A. Coburn, The $C^{*}$-algebra generated by an isometry I, Bull. Amer. Math. Soc. 13 (1967), 722-726.

8. R. G. Douglas, On the $C^{*}$-algebra of a one-parameter semigroup of isometries, Acta Math. 128 (1972), 143-152. 706.

9. S. Echterhoff, On induced covariant systems, Proc. Amer. Math. Soc. 108 (1990), $703-$

10. M. Laca and I. Raeburn, Semigroup crossed products and the Toeplitz algebras of nonabelian groups, J. Functional Analysis 139 (1996), 415-440.

11. J. Lorch and Q. Xu, Quasi-lattice ordered groups and Toeplitz algebras, J. Operator Theory 50 (2003), 221-247.

12. G. J. Murphy, Ordered groups and Toeplitz algebras, J. Operator Theory 18 (1987), 303-326.

13. A. Nica, $C^{*}$-algebras generated by isometries and Wiener-Hopf operators, J. Operator Theory 27 (1992), 17-52.

14. I. Raeburn and D. P. Williams, Morita equivalence and continuous-trace $C^{*}$-algebras, Math. Surveys and Monographs, Vol. 60 (Amer. Math. Soc., Providence, 1998). 